\title{
Language use affects food behaviours and food values among Mexican-origin adults in the USA
}

\author{
Brent A Langellier ${ }^{1, *}$, Ron Brookmeyer ${ }^{2}$, May C Wang ${ }^{3}$ and Deborah Glik ${ }^{3}$ \\ 'Division of Health Promotion Sciences, Mel \& Enid Zuckerman College of Public Health, University of Arizona, \\ PO Box 245209, Tucson, AZ 85724, USA: ${ }^{2}$ Department of Biostatistics, Fielding School of Public Health, \\ University of California, Los Angeles, Los Angeles, CA, USA: ${ }^{3}$ Department of Community Health Sciences, \\ Fielding School of Public Health, University of California, Los Angeles, Los Angeles, CA, USA
}

Submitted 22 August 2013: Final revision received 9 December 2013: Accepted 5 February 2014: First published online 3 April 2014

\begin{abstract}
Objective: Previous studies have established that acculturation is associated with dietary intake among Mexican immigrants and their offspring, but few studies have investigated whether food purchasing, food preparation or food-related values act as mechanisms of dietary acculturation. We examine the relationship between language use and a wide range of food behaviours and food-related values among Mexican-American adults.

Design: Nationally representative probability sample of the US population.

Setting: 2005-2010 National Health and Nutrition Examination Survey.

Subjects: Mexican-American adults ( $n$ 2792) at least 20 years of age.

Results: Mexican Americans who speak only or mostly English consume more energy from fast-food and sit-down restaurants and report increased consumption of non-homemade meals, fast-food and pizza meals, frozen meals and ready-to-eat meals relative to Spanish speakers. English speakers prepare one fewer homemade dinner per week and spend less time on meal preparation. English speakers are more likely than Spanish speakers to cite convenience as an important reason why they prefer fast food over cooking at home. There is no relationship between language use and the perceived importance of the nutritional quality, price or taste of fast food.

Conclusions: Our results provide evidence that the well-documented relationship between acculturation and diet among Mexican Americans may be just one indicator of a broader pattern characterized by decreased home meal preparation and increased reliance on convenience foods.
\end{abstract}

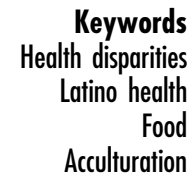

Over the last several decades, researchers have observed that Mexican Americans have paradoxically good health outcomes relative to their low socio-economic positions ${ }^{(1)}$. This health advantage, which has been observed for all-cause mortality, cancer, CVD and several other chronic conditions, is strongest in the foreign-born and deteriorates as immigrants spend increased time in the USA ${ }^{(1-5)}$. A common explanation is that acculturation, or exposure to and adoption of US culture, may have a negative effect on the health behaviours of immigrants and their offspring $^{(4,6,7)}$.

The relationship between acculturation and diet has received particular attention, perhaps because obesity rates are even higher among Mexican Americans than the general US population ${ }^{(8)}$. US-born and more-acculturated Mexican Americans consume fewer 'traditionally Mexican' food items (beans, tortillas, tortas, etc.) than their foreignborn and less-acculturated counterparts ${ }^{(9,10)}$. Studies have documented both healthy and unhealthy differences in diet, but the general consensus is that acculturation is associated with an overall decline in dietary quality ${ }^{(9,11-16)}$. For example, using bi-national data from the USA and Mexico, Batis et al. found that populations with greater exposure to the USA demonstrate decreased consumption of fruits and vegetables but increased consumption of several unhealthy foods and nutrients, including saturated fat, sugar, dessert and salty snacks, pizza and French fries ${ }^{(9)}$.

Several questions remain regarding how and why exposure to US culture affects Mexican Americans' food behaviours. An important but understudied topic is whether food purchasing and preparation behaviours vary based on nativity, language use or other measures of exposure and adoption of US culture ${ }^{(8,17,18)}$. The few studies conducted in this area have focused primarily on consumption of fast foods and sugar-sweetened beverages, both of which increase with acculturation ${ }^{(17,19,20)}$. We agree with previous conceptualizations of the dietary acculturation process, which posit that acculturation can 
act through a number of psychosocial, structural and economic pathways that affect where people shop for food, what types of food they purchase and how their food is prepared ${ }^{(8,17)}$. For example, recent immigrants may retain patterns of food behaviour established in their countries of origin, shop at different food outlets than their native-born counterparts, make different food choices in food stores and restaurants, or use more or less healthful preparation techniques. Language proficiency may also be an important determinant of food purchasing and preparation, because Spanish speakers might feel uncomfortable shopping at food outlets where English is dominant, have lower comprehension of food advertising, food packaging and nutrition labels, and have reduced exposure to other elements of US food culture. Understanding the relationship between acculturation and food purchasing and preparation is important because it may help explain variation in diet within the Mexican-American population as well as why obesity rates tend to increase within and across immigrant generations.

In the present study, we use data from Mexican-American adults who participated in the 2005-2010 National Health and Nutrition Examination Survey (NHANES) to assess the relationship between language use, nativity and food behaviours among Mexican-origin populations. Rather than focus exclusively on diet, we examine a broad range of food purchasing, preparation and consumption behaviours. We also examine whether any observed relationship between language use, nativity and food behaviours is explained by variation in educational attainment, socio-economic status and other sociodemographic characteristics. To understand psychosocial pathways through which exposure to US culture might affect the food behaviours of Mexican-origin populations, we examine reasons why participants prefer fast food to home meal preparation, as well as values that affect food choices in the supermarket.

\section{Methods}

\section{Data source}

Data for the current study are from public use data files of the 2005-2010 NHANES. Briefly, NHANES is a continuous series of annual studies that has been conducted since 1999 and is designed to assess the health and nutritional status of adults and children in the USA. NHANES uses a complex, multistage sampling design to obtain a sample representative of the non-institutionalized US population of all ages. Oversampling is used to produce sub-samples representative of national populations of children and adolescents, people living in low-income households and racial/ethnic minorities, including Mexican Americans.

NHANES includes both an in-person interview and a physical examination component. The interview contains demographic, dietary and health-related questions, while the examination includes medical, dental and physiological measurements taken by highly trained personnel. Participants also undergo two $24 \mathrm{~h}$ dietary recalls. In 2007, a Flexible Consumer Behavior Survey module was added to collect information on people's knowledge, attitudes and beliefs towards nutrition and food choices. Further details regarding the NHANES sampling strategy, study design and questionnaires are available elsewhere ${ }^{(21)}$.

\section{Analytic sub-sample}

In the present study we restrict our analyses to a subsample of 2792 Mexican-American adult participants in the 2005-2010 NHANES aged 20 years or older, including 1353 males and 1439 females. Of the 31034 total participants in these years of the survey, we exclude 13902 (45\%) because they were less than 20 years old and an additional 13956 (45\%) because they did not self-identify as Mexican American. Of the remaining 3176 Mexican-American adults, we exclude 384 who lacked data regarding their marital status ( $n$ 2), education ( $n$ 8), income ( $n$ 369) or language use $(n 5)$.

\section{Measurements}

\section{Language use}

We use participants' language use as a proxy for adoption of US culture, based on a question that asks, "What language do you usually speak at home?' Response options include: 'only Spanish', 'more Spanish than English', 'both equally', 'more English than Spanish' and 'only English'.

\section{Food purchasing, preparation and consumption}

NHANES participants are asked about a wide range of food purchasing, preparation and consumption behaviours. In the present study, we examine the relationship between language use and the following food behaviours: (i) participants' daily total energy intake, measured in $\mathrm{kJ} / \mathrm{d}$ based on two $24 \mathrm{~h}$ dietary recalls; (ii) participants' daily total energy intake from fast-food restaurants; (iii) the number of non-homemade and fast-food/pizza meals participants consumed in the previous week; (iv) the number of readyto-eat (i.e. supermarket deli or pre-packaged) and frozen meals participants consumed in the previous $30 \mathrm{~d}$; (v) the number of days that participants or another household member prepared dinner in the previous week; (vi) the number of minutes per day that participants or other household members spent to cook dinner and clean up; (vii) family spending on food in stores and restaurants in the previous $30 \mathrm{~d}$ (measured in \$US); and (viii) values related to preference of fast food $v$. home meal preparation and food choices in supermarkets. To assess fast food values, participants are asked whether or not they prefer fast food $v$. cooking at home because it is cheaper, more nutritious, tastes better, is more convenient or allows them to socialize. To assess values related to food choice in supermarkets, participants are asked about the importance of the following characteristics when they choose between 
foods at the supermarket: price, nutrition, taste, ease of preparation and how well the food keeps. Response options include: 'very important', 'somewhat important', 'not too important' and 'not at all important'.

\section{Statistical analyses}

We present the means and standard deviations of all continuous variables and percentage distributions of categorical variables. We use conditional means and cross-tabulation to assess whether food behaviour outcomes vary across language-use strata. We use $t$ tests to assess the statistical significance of these relationships ${ }^{(22)}$. We use multivariate logistic regression to examine whether any observed relationship between language use and food behaviour outcomes is explained by differences in sociodemographic characteristics between English- and Spanish-speaking participants. We examine the following three outcomes using multivariate logistic regression: (i) whether participants ate any food from a fast-food or sit-down restaurant during either of two days assessed via two $24 \mathrm{~h}$ dietary recalls; (ii) whether participants and their family members prepare dinner seven days per week as opposed to six days per week or less; and (iii) whether participants consumed two or more meals prepared outside the home in the previous week. We dichotomize the latter two outcomes (i.e. dinner preparation and prepared meal consumption) because they are each highly skewed and violate the normality assumption of linear regression. In particular, $60 \%$ of participants in our sample reported preparing dinners seven days per week and one-quarter reported consuming zero prepared meals in the previous week. The distribution was such that standard transformations (i.e. the square or square root, log transformation, etc.) did not result in normality.

We use multivariate linear regression to examine the following three outcomes: (i) the square root of restaurant dietary intake, measured in $\mathrm{kJ} / \mathrm{d}$, among participants who ate at a fast-food or sit-down restaurant during at least one of the two days measured via $24 \mathrm{~h}$ dietary recalls; (ii) the square root of expenditures on food in all stores during the previous month (\$US); and (iii) the square root of expenditures on restaurant food in the previous month (\$US). These outcomes are each right tailed and clearly violate the normality assumptions of linear regression; we use the square root to achieve a more normal distribution. For each outcome, we include an unadjusted model and a model adjusted for nativity, gender, age, marital status, educational attainment, family income and family size. For all analyses, we use sample weights included in the NHANES public use files that account for non-response, the complex design of the study and post-stratification ${ }^{(23)}$.

\section{Results}

In Table 1, we provide sociodemographic information regarding the 2792 Mexican-American adult participants in the 2005-2010 NHANES. Four in ten speak only Spanish in

Table 1 Sociodemographic characteristics by language use among Mexican-origin adults in the 2005-2010 National Health and Nutrition Examination Survey ( $n$ 2792)

\begin{tabular}{|c|c|c|c|c|c|c|c|}
\hline & \multicolumn{5}{|c|}{ Language use } & \multirow[b]{2}{*}{ Total } & \multirow[b]{2}{*}{$P$ value } \\
\hline & Only Spanish & More Spanish & Both & More English & Only English & & \\
\hline Gender (\%) & & & & & & & 0.0126 \\
\hline Male & $54 \cdot 7$ & $59 \cdot 5$ & $49 \cdot 9$ & $53 \cdot 3$ & $46 \cdot 6$ & $53 \cdot 3$ & \\
\hline Female & $45 \cdot 3$ & $40 \cdot 6$ & $50 \cdot 2$ & $46 \cdot 7$ & $53 \cdot 4$ & $46 \cdot 7$ & \\
\hline \multicolumn{8}{|l|}{ Age (years) } \\
\hline Mean & $40 \cdot 2$ & $38 \cdot 8$ & $39 \cdot 5$ & $39 \cdot 2$ & $39 \cdot 6$ & $39 \cdot 7$ & 0.56 \\
\hline SE & $1 \cdot 06$ & $0 \cdot 84$ & $1 \cdot 22$ & 0.96 & 0.72 & 0.53 & \\
\hline Marital status (\%) & & & & & & & $<0.001$ \\
\hline Married/cohabiting & $71 \cdot 8$ & $76 \cdot 0$ & $59 \cdot 0$ & $61 \cdot 6$ & $58 \cdot 1$ & $67 \cdot 2$ & \\
\hline Never married & $14 \cdot 6$ & $13 \cdot 2$ & $21 \cdot 6$ & $20 \cdot 7$ & $23 \cdot 6$ & $17 \cdot 6$ & \\
\hline Divorced/widowed/separated & $13 \cdot 6$ & $10 \cdot 8$ & $19 \cdot 5$ & $17 \cdot 8$ & $18 \cdot 4$ & $15 \cdot 3$ & \\
\hline Educational attainment (\%) & & & & & & & $<0.001$ \\
\hline$<9$ th grade & $54 \cdot 9$ & $26 \cdot 3$ & $13 \cdot 0$ & $5 \cdot 5$ & $3 \cdot 7$ & $30 \cdot 3$ & \\
\hline Some high school & $22 \cdot 1$ & $27 \cdot 7$ & $21 \cdot 1$ & $20 \cdot 3$ & $14 \cdot 9$ & $21 \cdot 4$ & \\
\hline High-school graduate & $13 \cdot 7$ & $21 \cdot 0$ & $29 \cdot 5$ & $25 \cdot 5$ & $24 \cdot 8$ & $20 \cdot 2$ & \\
\hline Some college/associate's degree & $7 \cdot 1$ & $17 \cdot 2$ & $30 \cdot 9$ & $31 \cdot 4$ & $38 \cdot 2$ & $20 \cdot 0$ & \\
\hline College graduate & $2 \cdot 1$ & $7 \cdot 9$ & $5 \cdot 6$ & $17 \cdot 2$ & $18 \cdot 4$ & $8 \cdot 1$ & \\
\hline \multicolumn{8}{|c|}{ Family income (\% of federal poverty level) } \\
\hline Mean & $1 \cdot 30$ & $1 \cdot 66$ & $2 \cdot 02$ & $2 \cdot 50$ & $2 \cdot 95$ & $1 \cdot 87$ & $<0.001$ \\
\hline SE & 0.04 & 0.07 & 0.09 & $0 \cdot 10$ & $0 \cdot 13$ & 0.05 & \\
\hline \multicolumn{8}{|l|}{ Family size } \\
\hline Mean & $4 \cdot 20$ & $4 \cdot 02$ & $4 \cdot 09$ & $3 \cdot 49$ & $3 \cdot 10$ & $3 \cdot 89$ & $<0.001$ \\
\hline SE & $0 \cdot 11$ & $0 \cdot 12$ & $0 \cdot 13$ & $0 \cdot 11$ & $0 \cdot 11$ & 0.07 & \\
\hline Nativity (\%) & & & & & & & $<0.001$ \\
\hline US-born & $3 \cdot 5$ & $22 \cdot 6$ & $59 \cdot 8$ & $83 \cdot 4$ & $93 \cdot 3$ & 38.9 & \\
\hline Foreign-born & $96 \cdot 5$ & $77 \cdot 4$ & $40 \cdot 2$ & $16 \cdot 6$ & $6 \cdot 7$ & $61 \cdot 2$ & \\
\hline
\end{tabular}

$P$ values are based on $\chi^{2}$ tests for categorical variables and $F$ tests for continuous variables. 
the home, 15\% speak more Spanish than English, 13\% speak both languages equally, 14\% speak more English than Spanish and 16\% speak only English. The sample is $47 \%$ female and $53 \%$ male, with a greater proportion of males in the Spanish-speaking strata $(P=0 \cdot 013)$. Mean age is 40 years, with a relatively equal age distribution across language-use strata. Family size and the proportion of participants who are married each decrease with English language use, while educational attainment and family income increase $(P<0 \cdot 001$ for all outcomes).

In Table 2, we present food purchasing, preparation and consumption behaviours by language use. Participants consume a mean of $8771 \mathrm{~kJ} / \mathrm{d}$ from all sources, with $1146 \mathrm{~kJ} / \mathrm{d}$ from fast-food restaurants and an additional $739 \mathrm{~kJ} / \mathrm{d}$ from sitdown restaurants. Total energy consumption is higher among participants who speak mostly Spanish $(P=0 \cdot 001)$ and mostly English $(P<0 \cdot 001)$ than Spanish-only speakers. Spanish-only speakers consume an average of $779 \mathrm{~kJ} / \mathrm{d}$ from fast food, compared with $1629 \mathrm{~kJ} / \mathrm{d}$ among those who speak both languages $(P<0 \cdot 001), 1749 \mathrm{~kJ} / \mathrm{d}$ among mostly-English speakers $(P<0 \cdot 001)$ and $1311 \mathrm{~kJ} / \mathrm{d}$ among English-only speakers $(P<0 \cdot 001)$. Similarly, Spanish-only speakers consume $461 \mathrm{~kJ} / \mathrm{d}$ from other restaurants while every other group consumes over $800 \mathrm{~kJ} / \mathrm{d}$ from these sources $(P<0 \cdot 05$ for all groups).

Data in Table 2 suggest that consumption of nonhomemade meals, fast-food meals, ready-to-eat meals and frozen meals each increase with English language use. For example, Spanish-only participants consume a mean of 2.5 non-homemade meals in the previous week, compared with 4.7 among mostly-English speakers $(P<0.001)$ and 4.5 among English-only speakers $(P<0 \cdot 001)$. Similarly, Spanish-only participants consume $1 \cdot 2$ fast-food and pizza meals in the previous week, compared with $2 \cdot 8$ and $2 \cdot 2$ among the mostly English and English only, respectively $(P<0 \cdot 001$ for both groups). Consumption of frozen meals, in particular, seems to increase with language use: Spanishonly participants consumed a mean of 0.5 frozen meals in the previous $30 \mathrm{~d}$, compared with $2 \cdot 6$ among mostly-English speakers $(P=0.001)$ and 3.5 among English-only speakers $(P<0 \cdot 001)$.

Frequency of dinner preparation and time spent to cook dinner and clean up both decrease with English language use. Participants who speak only Spanish cook dinner $6 \cdot 1$ times/week and spend $94 \mathrm{~min} /$ dinner cooking and cleaning up, compared with $5 \cdot 1$ dinners/week and $71 \mathrm{~min} /$ dinner among those who speak only English $(P<0 \cdot 001$ for both outcomes). There is no clear relationship between language use and the weekly number of meals families eat together. In the $30 \mathrm{~d}$ prior to the study, Spanish-only speakers spent more money than English-only speakers at all food stores (\$US $543 v$. \$US 421; $P=0 \cdot 001$ ) and supermarkets (\$US $450 v$. \$US 369; $P=0 \cdot 004$ ), but less at restaurants (\$US $132 v$. \$US 217; $P=0 \cdot 001$ ).

Figure 1 includes a series of bar charts indicating reasons participants might prefer eating at fast-food restaurants $v$. cooking at home (top panel) and the perceived

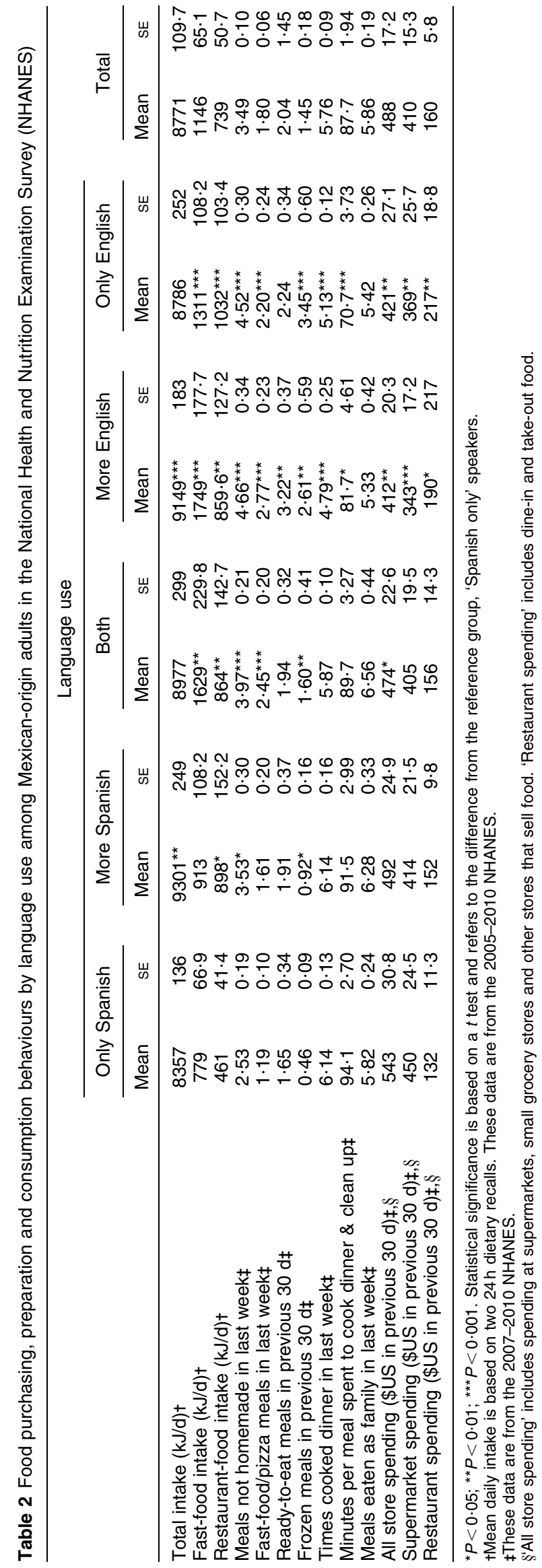




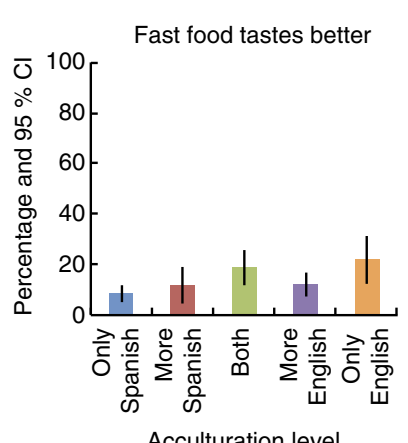

Acculturation level

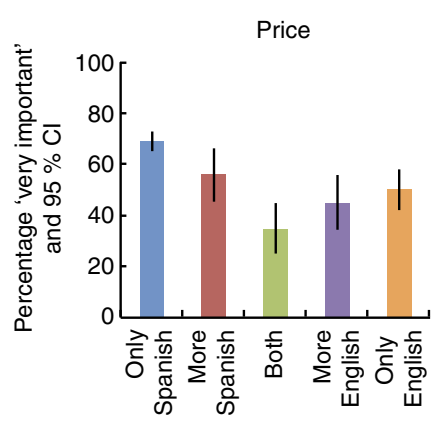

Acculturation level

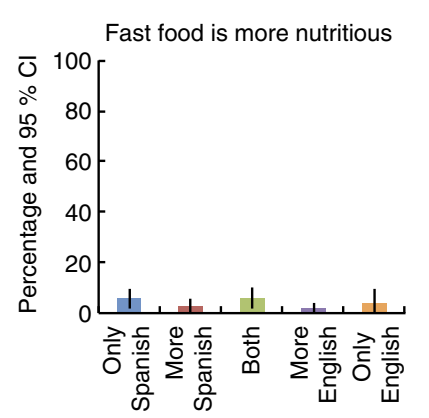

Acculturation level

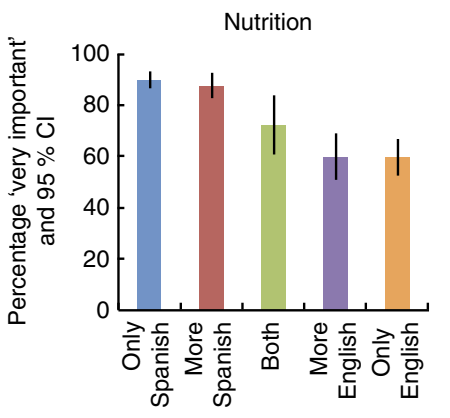

Acculturation level

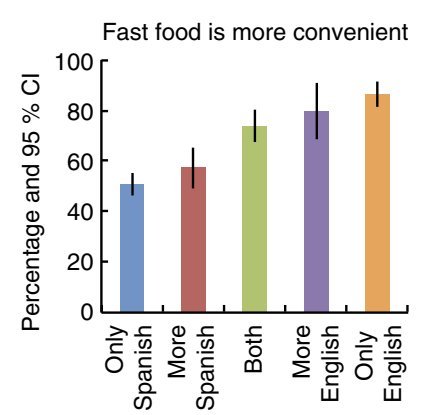

Acculturation level Fast food ( $n$ 1132)

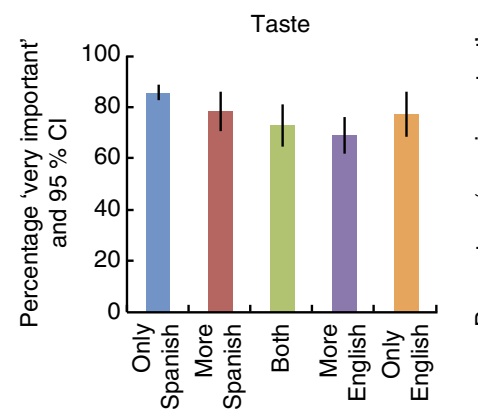

Acculturation level

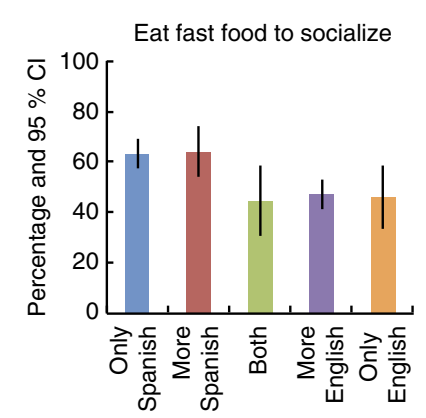

Acculturation level

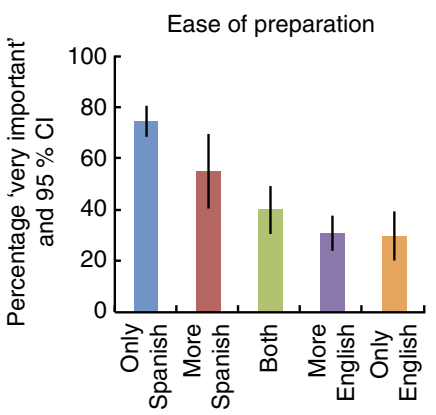

Acculturation level

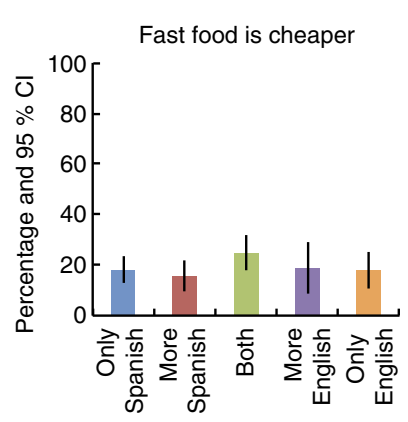

Acculturation level

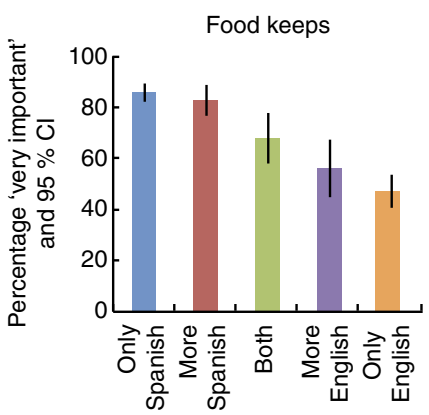

Acculturation level

Fig. 1 (colour online) Values related to fast-food purchasing (top panel) and food choices in supermarkets (bottom panel) by language use among Mexican-American adult participants in the 2007-2010 National Health and Nutrition Examination Survey. Values are percentages with their $95 \%$ confidence intervals represented by vertical bars 
Table 3 Unadjusted and adjusted logistic regression models predicting food behaviours among Mexican-origin adults in the National Health and Nutrition Examination Survey (NHANES)

\begin{tabular}{|c|c|c|c|c|c|c|c|c|c|c|c|c|}
\hline & \multicolumn{4}{|c|}{ Any fast-food/restaurant-food consumptiont } & \multicolumn{4}{|c|}{ Frequent dinner preparation $\ddagger$} & \multicolumn{4}{|c|}{ Frequent prepared meal consumption‡ } \\
\hline & \multicolumn{2}{|c|}{ (1) } & \multicolumn{2}{|c|}{$(2)$} & \multicolumn{2}{|c|}{ (3) } & \multicolumn{2}{|c|}{$(4)$} & \multicolumn{2}{|c|}{$(5)$} & \multicolumn{2}{|c|}{ (6) } \\
\hline & OR & SE & AOR & SE & OR & SE & AOR & SE & OR & SE & AOR & SE \\
\hline \multicolumn{13}{|l|}{ Language use } \\
\hline Only Spanish & \multicolumn{2}{|c|}{ Ref. } & \multicolumn{2}{|c|}{ Ref. } & \multicolumn{2}{|c|}{ Ref. } & \multicolumn{2}{|c|}{ Ref. } & \multicolumn{2}{|c|}{ Ref. } & \multicolumn{2}{|c|}{ Ref. } \\
\hline Mostly Spanish & $1 \cdot 555^{\star}$ & 0.257 & $1 \cdot 276$ & $0 \cdot 221$ & $0 \cdot 708$ & $0 \cdot 146$ & 0.951 & $0 \cdot 197$ & $1 \cdot 919^{\star \star \star}$ & $0 \cdot 260$ & $1 \cdot 485^{\star}$ & 0.232 \\
\hline Both equally & $2 \cdot 967^{\star \star \star}$ & 0.583 & $1.996^{\star \star}$ & $0 \cdot 410$ & $0.545^{\star \star}$ & 0.0925 & $0 \cdot 840$ & $0 \cdot 165$ & $2 \cdot 307^{\star \star *}$ & 0.331 & $1 \cdot 517^{\star}$ & 0.267 \\
\hline Mostly English & $4 \cdot 263^{\star \star \star}$ & $0 \cdot 800$ & $2 \cdot 579^{\star \star \star}$ & 0.597 & $0 \cdot 225^{\star \star \star}$ & 0.0392 & $0 \cdot 460^{\star \star}$ & $0 \cdot 105$ & $3 \cdot 739^{\star \star \star}$ & $1 \cdot 009$ & $1.998^{*}$ & 0.623 \\
\hline Only English & $2 \cdot 889^{\star \star \star}$ & 0.634 & $1 \cdot 615^{\star}$ & 0.374 & $0 \cdot 213^{\star * *}$ & 0.0406 & $0.570^{*}$ & $0 \cdot 147$ & $4 \cdot 228^{\star \star *}$ & 0.843 & $1 \cdot 822^{*}$ & 0.528 \\
\hline Foreign-born & & & $0 \cdot 839$ & $0 \cdot 136$ & & & $1 \cdot 404^{*}$ & $0 \cdot 189$ & & & $0.669^{*}$ & $0 \cdot 116$ \\
\hline Male & & & $1 \cdot 126$ & $0 \cdot 123$ & & & $0 \cdot 840$ & 0.0902 & & & $1 \cdot 689^{\star \star \star}$ & $0 \cdot 138$ \\
\hline Age (years) & & & $0.980^{\star \star \star}$ & 0.00452 & & & $1 \cdot 016^{\star \star}$ & 0.00501 & & & $0.971^{\star \star \star}$ & 0.00407 \\
\hline \multicolumn{13}{|l|}{ Marital status } \\
\hline Married/cohabiting & & & \multicolumn{2}{|c|}{ Ref. } & \multicolumn{4}{|c|}{ Ref. } & & & \multicolumn{2}{|c|}{ Ref. } \\
\hline Never married & & & $1 \cdot 255$ & 0.239 & & & $1 \cdot 199$ & $0 \cdot 170$ & & & $1 \cdot 119$ & 0.218 \\
\hline Divorced/widowed/separated & & & $1 \cdot 169$ & $0 \cdot 181$ & & & 0.771 & $0 \cdot 118$ & & & $1 \cdot 281$ & 0.203 \\
\hline \multicolumn{13}{|l|}{ Educational attainment } \\
\hline$<9$ th grade & & & \multicolumn{2}{|c|}{ Ref. } & \multicolumn{4}{|c|}{ Ref. } & & & \multicolumn{2}{|c|}{ Ref. } \\
\hline Some high school & & & $1 \cdot 231$ & $0 \cdot 217$ & & & 0.978 & $0 \cdot 210$ & & & $0 \cdot 848$ & $0 \cdot 217$ \\
\hline High-school graduate & & & $1 \cdot 300$ & 0.244 & & & 0.839 & $0 \cdot 208$ & & & $1 \cdot 137$ & $0 \cdot 191$ \\
\hline Some college/associate's degree & & & $2 \cdot 016^{\star \star}$ & 0.433 & & & $0 \cdot 741$ & $0 \cdot 171$ & & & $1 \cdot 201$ & 0.250 \\
\hline College graduate & & & 0.968 & 0.302 & & & 0.609 & $0 \cdot 174$ & & & 1.528 & 0.450 \\
\hline Family income ( $\%$ of federal poverty level) & & & $1 \cdot 113$ & 0.0634 & & & $0 \cdot 753^{\star \star *}$ & 0.0477 & & & $1 \cdot 296^{\star \star *}$ & 0.0652 \\
\hline Family size & \multirow{2}{*}{\multicolumn{4}{|c|}{$\begin{array}{c}0.960 \\
2572\end{array}$}} & & & $1 \cdot 162^{\star \star}$ & 0.0568 & & & $0 \cdot 990$ & 0.0313 \\
\hline$n$ & & & & & \multicolumn{4}{|c|}{1861} & \multicolumn{4}{|c|}{1871} \\
\hline
\end{tabular}

AOR, adjusted odds ratio; Ref., reference category.

'Any fast-food/restaurant-food consumption' defined as any food item during either of two days measured via $24 \mathrm{~h}$ dietary recalls. 'Frequent dinner preparation' defined as having prepared seven dinners in the previous week. 'Frequent prepared meal consumption' defined as two or more meals prepared outside the home in the previous week.

${ }^{\star} P<0.05,{ }^{\star \star} P<0.01,{ }^{\star \star \star} P<0.001$

tThese data are from the 2005-2010 NHANES.

$\ddagger$ These data are from the 2007-2010 NHANES. 
importance of food characteristics when choosing between items at the supermarket (bottom panel). The data do not reveal a clear relationship between language use and perceived importance of taste, nutrition or price as reasons participants prefer fast food to cooking at home. In contrast, $63 \%$ of Spanish-only participants said that the ability to socialize was a very important reason they might prefer fast food over home cooked meals, compared with $45-47 \%$ of participants who speak both languages, mostly English or only English. Furthermore, the data suggest a clear relationship between language use and the perceived importance of the convenience of fast food: half of Spanish-only participants cited convenience as a very important reason to prefer fast food, compared with $86 \%$ of participants who speak mostly English or English only.

The data suggest that participants perceive taste to be very important when choosing between food items at the supermarket, regardless of language use. Spanish-speaking participants perceive every other perceived value to be more important than their more-acculturated counterparts. For example, about seven in ten participants who speak only Spanish perceive price as very important, compared with $45 \%$ and $50 \%$ of those who speak mostly English or only English, respectively. Nine in ten participants who speak mostly Spanish or only Spanish perceive nutrition as very important, compared with two-thirds of those who speak only English. Three in four Spanish-only participants perceive preparation ease as very important, compared with $30 \%$ of those who speak mostly English or only English.

Table 3 displays the results of logistic regression models predicting the following: (i) whether participants ate any food from a fast-food or sit-down restaurant during either of two days assessed via two $24 \mathrm{~h}$ dietary recalls; (ii) whether participants and their family members prepare dinner seven days per week as opposed to six days per week or less; and (iii) whether participants consumed two or more meals prepared outside the home in the previous week. Unadjusted for other factors, English-only speakers had nearly three times the odds of eating at a restaurant as Spanish-only speakers. Adjustment for sociodemographic factors attenuates this relationship somewhat, but there are still large and significant differences across languageuse strata. The adjusted model suggests that few other sociodemographic factors are associated with restaurant consumption among Mexican Americans, with the primary exception being a negative association with age $(P<0 \cdot 001)$. Unadjusted for other factors, the odds of frequent dinner preparation are lower among English speakers. This relationship is attenuated by adjustment for sociodemographic factors, but Spanish-only participants still have significantly greater odds of being 'frequent' dinner preparers relative to their mostly-English $(P<0 \cdot 01)$ and only-English $(P<0 \cdot 05)$ counterparts. The foreign-born also have greater odds of being frequent dinner preparers (adjusted $\mathrm{OR}=1 \cdot 4$; $P<0 \cdot 05$ ), which is important since most Spanish-speaking participants are also foreign-born. Our regression models predicting frequent consumption of prepared meals reveal a similar pattern relative to the two previous food behaviour outcomes: unadjusted for other factors, English-speaking participants are significantly more likely to frequently consume prepared foods. This relationship is somewhat attenuated by adjustment for sociodemographic factors, but significant differences remain between English speakers and Spanish speakers.

In Table 4, we present three sets of linear regression models. The first set of models predicts the square root of energy intake from restaurants $(\mathrm{kJ} / \mathrm{d})$ among participants who ate at a fast-food or sit-down restaurant during at least one of the two days measured via $24 \mathrm{~h}$ dietary recalls. Unadjusted for other factors, participants who speak Spanish and English equally, mostly English or only English consume more energy at restaurants than participants who speak Spanish only $(P<0.05$ in all cases). This relationship is greatly reduced and all point estimates become insignificant after adjustment for sociodemographic factors. The adjusted model suggests that the foreign-born consume less energy at restaurants than the US-born $(P<0 \cdot 01)$. The second set of models in Table 4 indicates that, unadjusted for other factors, food expenditures at supermarkets and other stores are negatively associated with English language use. This relationship is attenuated by adjustment for sociodemographic factors, but spending is still significantly greater among participants who speak English and Spanish equally $(P<0 \cdot 01)$ and mostly English $(P<0 \cdot 01)$ relative to those who speak Spanish only. The foreign-born have significantly greater food expenditures $(P<0 \cdot 05)$, which amplifies the difference between Spanish speakers, who are predominantly foreign-born, and English speakers, who are predominantly US-born. The final set of models suggests that, without adjustment for other factors, English speakers spend more money at restaurants than Spanish speakers. This relationship is explained by sociodemographic differences between language-use strata. Younger age, greater family income and larger family size are positively associated with restaurant expenditures.

\section{Discussion}

We believe that the present study has provided new insight into the relationship between language use and food behaviours among the Mexican-origin population in the USA. Previous research has documented differences between more- and less-acculturated Latinos in consumption of specific foods and nutrients, as well as found increased purchasing and consumption of fast food among the more acculturated ${ }^{(9,11-17,19)}$. Consistent with this research, we found that purchasing and consumption of fast food and other restaurant food increased with English language use ${ }^{(17,19)}$. We also found that increased reliance on restaurant food is part of a larger pattern 
Table 4 Unadjusted and adjusted linear regression models predicting food behaviours among Mexican-origin adults in the National Health and Nutrition Examination Survey (NHANES) Square root of energy intake from restaurants $(\mathrm{kJ} / \mathrm{d})+\quad$ Square root of all store spending $(\$ \cup S) \ddagger \quad$ Square root of restaurant spending $(\$ \cup S) \ddagger$

\begin{tabular}{|c|c|c|c|c|c|c|c|c|c|c|}
\hline \multicolumn{2}{|c|}{ (1) } & \multicolumn{2}{|c|}{ (2) } & \multicolumn{2}{|c|}{ (3) } & \multicolumn{2}{|c|}{ (4) } & \multicolumn{2}{|c|}{ (5) } & (6) \\
\hline$b$ & SE & $b$ & SE & $b$ & SE & $b$ & SE & $b$ & SE & SE \\
\hline
\end{tabular}

\begin{tabular}{|c|c|c|c|c|c|c|c|c|c|c|c|c|}
\hline \multicolumn{13}{|l|}{ Lanquage use } \\
\hline Only Spanish & \multicolumn{2}{|c|}{ Ref. } & \multicolumn{2}{|c|}{ Ref. } & \multicolumn{2}{|l|}{ Ref. } & \multicolumn{2}{|c|}{ Ref. } & \multicolumn{2}{|c|}{ Ref. } & \multicolumn{2}{|c|}{ Ref. } \\
\hline Mostly Spanish & 4.022 & 2.094 & $1 \cdot 404$ & $2 \cdot 220$ & -0.877 & 0.805 & -0.566 & 0.712 & $1 \cdot 403^{*}$ & 0.573 & 0.498 & 0.568 \\
\hline Both equally & $7 \cdot 837^{* *}$ & $2 \cdot 830$ & $2 \cdot 711$ & $2 \cdot 602$ & $-1 \cdot 679^{\star}$ & 0.640 & $-1 \cdot 685^{\star *}$ & 0.525 & $1.504^{*}$ & 0.623 & $-0 \cdot 137$ & 0.616 \\
\hline Mostly English & $5 \cdot 276^{\star}$ & $2 \cdot 199$ & -0.975 & 2.792 & $-2 \cdot 868^{\star \star \star}$ & 0.704 & $-1 \cdot 834^{\star *}$ & 0.636 & $3 \cdot 123^{\star \star \star}$ & 0.782 & 0.826 & 0.556 \\
\hline Only English & $5 \cdot 716^{* *}$ & $1 \cdot 775$ & $-0 \cdot 106$ & $3 \cdot 411$ & $-2 \cdot 392^{\star \star \star}$ & 0.644 & -1.058 & 0.553 & $3.964^{\star \star *}$ & 0.667 & 0.698 & 0.677 \\
\hline Foreign-born & & & $-5.032^{\star}$ & 2.092 & & & $0.736^{*}$ & 0.335 & & & -0.265 & 0.422 \\
\hline Male & & & $7 \cdot 506^{\star \star *}$ & $1 \cdot 162$ & & & 0.423 & $0 \cdot 287$ & & & $0.569^{\star}$ & 0.250 \\
\hline Age (years) & & & $-0.344^{\star \star *}$ & 0.0522 & & & -0.00940 & 0.0110 & & & $-0.0553^{\star \star \star}$ & 0.0103 \\
\hline \multicolumn{13}{|l|}{ Marital status } \\
\hline Married/cohabiting & & & \multicolumn{2}{|c|}{ Ref. } & \multicolumn{4}{|c|}{ Ref. } & & & \multicolumn{2}{|c|}{ Ref. } \\
\hline Never married & & & $2 \cdot 614$ & $1 \cdot 879$ & & & 0.447 & $0 \cdot 420$ & & & 0.838 & 0.681 \\
\hline Divorced/widowed/separated & & & $2 \cdot 191$ & $1 \cdot 306$ & & & -0.439 & 0.405 & & & $0 \cdot 282$ & 0.529 \\
\hline \multicolumn{3}{|l|}{ Educational attainment } & \multirow{2}{*}{\multicolumn{2}{|c|}{ Ref. }} & \multirow{2}{*}{\multicolumn{4}{|c|}{ Ref. }} & & & \multirow{2}{*}{\multicolumn{2}{|c|}{ Ref. }} \\
\hline$<9$ th grade & & & & & & & & & & & & \\
\hline Some high school & & & $1 \cdot 227$ & $2 \cdot 161$ & & & 0.0677 & 0.539 & & & 0.289 & 0.825 \\
\hline High-school graduate & & & 0.387 & $2 \cdot 052$ & & & $-1 \cdot 222^{*}$ & 0.528 & & & 0.338 & 0.651 \\
\hline Some college/associate's degree & & & $2 \cdot 155$ & $2 \cdot 137$ & & & $-0 \cdot 725$ & $0 \cdot 461$ & & & 0.932 & 0.808 \\
\hline College graduate & & & $-1 \cdot 703$ & $2 \cdot 405$ & & & $-1 \cdot 321$ & $1 \cdot 047$ & & & 0.869 & 0.644 \\
\hline Family income (\% of federal poverty level) & & & 0.715 & 0.536 & & & $0.955^{\star \star *}$ & $0 \cdot 190$ & & & $1 \cdot 817^{\star \star \star}$ & $0 \cdot 161$ \\
\hline Family size & \multirow{2}{*}{\multicolumn{4}{|c|}{$1507^{-0.515}$}} & & & $1 \cdot 819^{\star \star \star}$ & $0 \cdot 154$ & & & $0.516^{\star \star}$ & $0 \cdot 164$ \\
\hline$n$ & & & & & \multicolumn{4}{|c|}{1826} & \multicolumn{4}{|c|}{1833} \\
\hline
\end{tabular}

Ref., reference category.

'Energy intake from restaurants' includes only participants who reported eating at a fast-food or sit-down restaurant during either of two days measured via $24 \mathrm{~h}$ dietary recalls. 'All store spending' includes spending at supermarkets, small grocery stores and other stores that sell food in the previous $30 \mathrm{~d}$. 'Restaurant spending' includes dine-in and take-out food in the previous $30 \mathrm{~d}$.

${ }^{\star} P<0.05,{ }^{\star \star} P<0.01,{ }^{* \star \star} P<0.001$

tThese data are from the 2005-2010 NHANES.

$\ddagger$ These data are from the 2007-2010 NHANES. 
characterized by less frequent home meal preparation and increased reliance on convenience foods among English speakers. English speakers spend more money at restaurants, less money at grocery stores and supermarkets, and consume more non-homemade meals of all types, including fast-food and pizza meals, frozen meals and ready-to-eat meals. These findings are troubling from a public health perspective because a wide body of research has documented that homemade meals tend to be more healthful than prepared foods ${ }^{(24-27)}$

Our data also suggest that food values vary based on participants' language use. In particular, English speakers were much more likely than Spanish speakers to report that convenience is a very important reason why they prefer fast food to cooking at home. This suggests that the relationship between language use and diet might be caused by differences not only in tastes and preferences, but also in motivation, time, ability or commitment to preparing meals at home. Furthermore, Spanish-speaking participants placed greater importance on price, nutrition, ease of preparation and how well foods keep when choosing between items at the grocery store or supermarket. This may suggest that, relative to their moreacculturated counterparts, Spanish speakers are more deliberate about what foods they purchase in order to stretch their food budgets, provide good nutrition to themselves and their families, and reduce the burden of home meal preparation. The relatively higher importance placed on food price, in particular, likely reflects the fact that native-born and English-speaking Latinos tend to have high incomes and presumably can spend more money on food ${ }^{(26)}$.

Importantly, we also found that at least some of the differences in food behaviours between English speakers and Spanish speakers are explained by differences in sociodemographic characteristics. In particular, large and significant differences across language-use strata in energy intake from restaurants and restaurant food expenditures were completely explained by sociodemographics. Differences between English- and Spanish speakers in the other food behaviours were attenuated by adjustment for sociodemographics, although significant differences persisted. Relative to English-speaking Mexican Americans and most other US sub-populations, Spanish speakers are more likely to be foreign-born, male, have larger families and have lower levels of income and education. Many of these characteristics affect food behaviours and other health outcomes, and can be thought of as the underlying cause of much of the difference between English- and Spanish speakers. Still, the fact that many differences in food behaviours were unexplained by sociodemographic factors suggests that immigrants and their offspring may be able to adapt to the US context while still maintaining some of the healthier food behaviours practised in Mexico.

We feel it is important to note that our study is based on the Mexican-American adult population in the USA during a specific time period, between 2005 and 2010, and that duration since migration is relatively high among immigrants in the sample (i.e. over $60 \%$ report time in the USA of $\geq 10$ years). Obesity and other diet-related chronic diseases have increased dramatically in Mexico, especially over the last decade, representing an enormous health and economic burden on the country ${ }^{(28)}$. Many of our participants migrated prior to these secular increases and, like the majority of Mexican immigrants, originated from rural contexts and low socio-economic strata where obesity rates were low compared with the rest of the country $^{(29,30)}$. As Mexico continues to undergo economic development and urbanization, unhealthy food behaviours and diet-related chronic diseases may continue to increase. As a result of these trends, the 'health advantage' of Mexican immigrants may be less pronounced in recent and future waves of immigrants than among the longertenured immigrants we observed in our study. As a result, the relationships we observed may change as immigrants arrive to the USA with less healthy behaviours.

The present study has a number of important limitations and strengths. NHANES data are cross-sectional, making it impossible to determine causality in the relationship between language use and food behaviours. Self-selection among Mexican Americans who chose to participate in NHANES also represents a potentially serious limitation of our study. In particular, undocumented immigrants are difficult to reach in survey research and have high refusal rates. Since undocumented immigrants represent a relatively large proportion of the foreign-born Mexican-American population, self-selection based on legal status could be a serious source of bias in our results. We posit that refusal rates among undocumented immigrants are likely similar between NHANES and other household surveys, since legal status is not among the inclusion criteria of the study. NHANES does not collect data regarding the legal status of participants, making the potential for this type of selection bias difficult to assess.

We used measures of language use and nativity to assess whether exposure to and adoption of US culture affects food behaviours, but these measures may be flawed. Previous research has established that acculturation is both a multidimensional and multidirectional process, whereby immigrants and their offspring can adopt the cultural patterns, practices and beliefs of their host country, retain cultural elements from their country of origin, or become multicultural ${ }^{(31)}$. Furthermore, immigrants and their offspring can undergo changes in one dimension of the acculturative process (e.g. language use) but remain unchanged with regard to other dimensions (e.g. cultural beliefs). We believe it is possible and likely that dimensions of the acculturative process other than language use could affect the food behaviours of Mexican-origin populations, but this is impossible to assess given the limitations of the NHANES data. Of note, NHANES also includes the time participants spent in the USA, which could 
be considered a good proxy for immigrants' exposure to US culture. As a sensitivity analysis, we used multivariate regression analyses (similar to those in Tables 3 and 4) to examine whether immigrants with varying time in the USA differed with regard to food behaviour outcomes. We found that the 'nativity effect' was generally the same regardless of time spent in the USA and did not explain the relationship between language use and food behaviours. For the sake of parsimony and to more clearly communicate the difference between US- and foreign-born participants, we chose not to include time spent in the USA in our final models. Similarly, the acculturation construct may be better measured with a multidimensional scale assessing friendship ties, media use, ethnic self-identification, and ethno-cultural attitudes, beliefs and practices, but this type of multidimensional scale is unavailable in NHANES ${ }^{(31,32)}$.

These limitations notwithstanding, we believe our study has important strengths and makes significant contributions to the existing literature in this field. Rather than focusing narrowly on the relationship between acculturation and diet, we have examined a broad set of food behaviours that ultimately determine what people eat. We believe this approach is a strength of our study, because behaviours such as where people shop for food, what they purchase, and if and how they prepare it are modifiable behaviours that can be targeted with interventions. This broad approach was greatly facilitated by our use of the NHANES data set, which contains a wealth of information regarding food purchasing, preparation and consumption. The NHANES data allowed us to examine novel aspects of Mexican Americans' food behaviours, particularly those related to food purchasing and preparation as well as food-related values. Another important strength is that NHANES oversamples among minority populations, which allowed us to study a large, representative sub-sample of the Mexican-American population.

\section{Conclusion}

In conclusion, the main finding of the present study is that the previously observed relationship between exposure to US culture and diet may be indicative of a larger pattern characterized by decreased home meal preparation in more-acculturated populations and increased reliance on convenience foods ${ }^{(9,11,17)}$. Our findings suggest that interventionists should concentrate on promoting home meal preparation or improving the nutritional quality of prepared, packaged and other convenience foods purchased by Mexican Americans. The latter strategy might include increasing the breadth, depth, quality, taste, prices and advertising of prepared healthy foods available in food outlets where Mexican Americans shop. This might be particularly effective because it would avoid the need to reduce consumption of convenience foods, but still allow for a range of healthy choices.

\section{Acknowledgements}

Sources of funding: This study was funded by the National Heart, Lung, and Blood Institute (grant numbers P50HL105188 and F31HL116109). The National Heart, Lung, and Blood Institute had no role in the design, analysis or writing of this article. Conflict of interest: None. Authors' contributions: B.A.L. conceptualized the study, conducted the statistical analysis and wrote the manuscript. R.B. assisted with the conceptualization and analyses and provided revisions for the manuscript. M.C.W. and D.G. assisted with the conceptualization and provided revisions for the manuscript. Ethics statement: The current study does not meet the definition of human subjects research, since our analyses were based on previously collected data without personally identifying information. Acknowledgements: The authors thank Anne Pebley and Jennie Brand for their substantial contributions to the conceptualization of this manuscript.

\section{References}

1. Markides KS \& Coreil J (1986) The health of Hispanics in the southwestern United States: an epidemiologic paradox. Public Health Rep 101, 253-265.

2. Sorlie PD, Backlund E, Johnson NJ et al. (1993) Mortality by Hispanic status in the United States. JAMA 270, 2464-2468.

3. Abraído-Lanza AF, Dohrenwend BP, Ng-Mak DS et al. (1999) The Latino mortality paradox: a test of the 'salmon bias' and healthy migrant hypotheses. Am J Public Health 89, 1543-1548.

4. Palloni A \& Arias E (2004) Paradox lost: explaining the Hispanic adult mortality advantage. Demography 41, 385-415.

5. Sundquist J \& Winkleby MA (1999) Cardiovascular risk factors in Mexican American adults: a transcultural analysis of NHANES III, 1988-1994. Am J Public Health 89, 723-730.

6. Abraido-Lanza AF, Armbrister AN, Florez KR et al. (2006) Toward a theory-driven model of acculturation in public health research. Am J Public Health 96, 1342-1346.

7. Abraído-Lanza AF, Chao MT \& Flórez KR (2005) Do healthy behaviors decline with greater acculturation? Implications for the Latino mortality paradox. Soc Sci Med 61, 1243-1255.

8. Satia-Abouta J (2003) Dietary acculturation: definition, process, assessment, and implications. Int J Hum Ecol 4, 71-86.

9. Batis C, Hernandez-Barrera L, Barquera S et al. (2011) Food acculturation drives dietary differences among Mexicans, Mexican Americans, and Non-Hispanic Whites. J Nutr 141, 1898-1906.

10. Winham DM \& Florian TA (2010) Hispanic women in EFNEP have low adherence with dietary guidelines regardless of acculturation level. J Hunger Environ Nutr 5, 498-509.

11. Ayala GX, Baquero B \& Klinger S (2008) A systematic review of the relationship between acculturation and diet among Latinos in the United States: implications for future research. J Am Diet Assoc 108, 1330-1344.

12. Duffey KJ, Gordon-Larsen P, Ayala GX et al. (2008) Birthplace is associated with more adverse dietary profiles for US-born than for foreign-born Latino adults. J Nutr 138, 2428-2435.

13. Gordon-Larsen P, Harris KM, Ward DS et al. (2003) Acculturation and overweight-related behaviors among Hispanic immigrants to the US: the National Longitudinal Study of Adolescent Health. Soc Sci Med 57, 2023-2034. 
14. Perez-Escamilla R (2009) Dietary quality among Latinos: is acculturation making us sick? J Am Diet Assoc 109, 988-991.

15. Perez-Escamilla R (2011) Acculturation, nutrition, and health disparities in Latinos. Am J Clin Nutr 93, issue 5, 1163S-1167S

16. Perez-Escamilla R \& Putnik P (2007) The role of acculturation in nutrition, lifestyle, and incidence of type 2 diabetes among Latinos. J Nutr 137, 860-870.

17. Ayala GX, Mueller K, Lopez-Madurga E et al. (2005) Restaurant and food shopping selections among Latino women in Southern California. J Am Diet Assoc 105, 38-45.

18. Akresh IR (2007) Dietary assimilation and health among Hispanic immigrants to the United States. J Health Soc Behav 48, 404-417.

19. Kasirye OC, Walsh JA, Romano PS et al. (2005) Acculturation and its association with health-risk behaviors in a rural Latina population. Ethn Dis 15, 733-739.

20. Sharkey JR, Johnson CM \& Dean WR (2011) Nativity is associated with sugar-sweetened beverage and fast-food meal consumption among Mexican-origin women in Texas border colonias. Nutr J 10, 101.

21. US Department of Health and Human Services, Centers for Disease Control and Prevention \& National Center for Health Statistics (2007) National Health and Nutrition Examination Survey, 2007-2008: Overview. Hyattsville, MD: US Department of Health and Human Services.

22. StataCorp (2011) Stata 12 Base Reference Manual. College Station, TX: StataCorp LP.

23. National Center for Health Statistics (2013) Continuous NHANES Web Tutorial: Specifying Weighting Parameters. http://www.cdc.gov/nchs/tutorials/nhanes/SurveyDesign/ Weighting/intro.htm (accessed April 2013).

24. Guthrie JF, Lin B-H \& Frazao E (2002) Role of food prepared away from home in the American diet, 1977-78 versus 1994-96: changes and consequences. J Nutr Educ Behav 34, 140-150.

25. Bassett MT, Dumanovsky T, Huang C et al. (2008) Purchasing behavior and calorie information at fast-food chains in New York City, 2007. Am J Public Health 98, 1457-1459.

26. Bowman SA \& Vinyard BT (2004) Fast food consumption of US adults: impact on energy and nutrient intakes and overweight status. J Am Coll Nutr 23, 163-168.

27. Lin BH, Guthrie J \& Frazao E (1999) Nutrient contribution of food away from home. In America's Eating Habits: Changes and Consequences. Agriculture Information Bulletin no. AIB-750, pp. 213-242 [E Frazao, editor]. Washington, DC: Economic Research Service, US Department of Agriculture.

28. Zallman L, Himmelstein DH, Woolhandler S et al. (2012) Undiagnosed and uncontrolled hypertension and hyperlipidemia among immigrants in the US. J Immigr Minor Health 15, 858-865.

29. Olaiz-Fernández G, Rivera-Dommarco J, Shamah-Levy T et al. (2006) Encuesta Nacional de Salud y Nutrición 2006. Cuernavaca, México: Instituto Nacional de Salud Pública.

30. Riosmena F \& Massey DS (2012) Pathways to El Norte: origins, destinations, and characteristics of Mexican migrants to the United States. Int Migr Rev 46, 3-36.

31. Marin G \& Gamba RJ (1996) A new measurement of acculturation for hispanics: the Bidimensional Acculturation Scale for Hispanics (BAS). Hispanic J Behav Sci 18, 297-316.

32. Cuellar I, Arnold B \& Maldonado R (1995) Acculturation rating scale for Mexican Americans-II: a revision of the original ARSMA Scale. Hispanic J Behav Sci 17, 275-304. 